\title{
Decomposing the Lattice of Meaningless Sets in the Infinitary Lambda Calculus
}

\author{
Paula Severi and Fer-Jan de Vries \\ Department of Computer Science, University of Leicester, UK
}

\begin{abstract}
The notion of a meaningless set has been defined for infinitary lambda calculus axiomatically. Standard examples of meaningless sets are sets of terms that have no head normal form, the set of terms without weak head normal form and the set of rootactive terms. The collection of meaningless sets is a lattice. In this paper, we study the way this lattices decompose as union of more elementary key intervals. We also analyse the distribution of the sets of meaningless terms in the lattice by selecting some sets as key vertices and study the cardinality in the intervals between key vertices. As an application, we prove that the lattice of meaningless sets is neither distributive nor modular. Interestingly, the example translates into a counterexample that the lattice of lambda theories is not modular.
\end{abstract}

\section{Introduction}

Classical, finite lambda calculus [1] considers only finite terms. It can not express inside the calculus that certain terms have an infinite normal form. For example, the term $M M$ where $M=\lambda x . f(x x)$ has the infinite normal form $f(f(f(\ldots)))$ which is the limit of the reduction sequence $M M \rightarrow_{\beta} f(M M) \rightarrow_{\beta}$ $f(f(M M)) \rightarrow_{\beta} \ldots$. Infinitary lambda calculus aims to treat finite and infinite terms in one notational framework with notation for finite and infinite reductions. It allows us to express that the above reduction sequence has the infinite term $f^{\omega}$ as limit. However, the natural extension of finite lambda calculus with infinite terms and infinite reductions ruins the confluence property [7]. For example, the term $N N$, where $N=\lambda x . \mathbf{I}(x x)$ and $\mathbf{I}=\lambda x . x$ reduces both to $\mathbf{I}^{\omega}$ and $\boldsymbol{\Omega}=(\lambda x . x x)(\lambda x . x x)$, which can only reduce to themselves and not be joined by even infinite reductions.

Needed to restore the confluence property $[7,6,8,5]$ is a designated set of meaningless terms (for short meaningless set), that is, a set satisfying the Axioms of Meaninglessness $[10,5]$ together with a new rewrite rule that allows any meaningless terms to be rewritten to a fresh symbol $\perp$. Those Axioms are general assumptions needed to prove confluence of the infinitary lambda calculus $[10,5]$. By changing the meaningless set, we obtain different notions of $\perp$-reduction and different infinite extensions. Each of these extensions is normalising and confluent, so that the set of its normal forms becomes a model of lambda calculus.

A standard example of a meaningless set is the set $\overline{\mathcal{H N}}$ of terms without head normal form. The normal forms of the corresponding infinitary extension of finite 


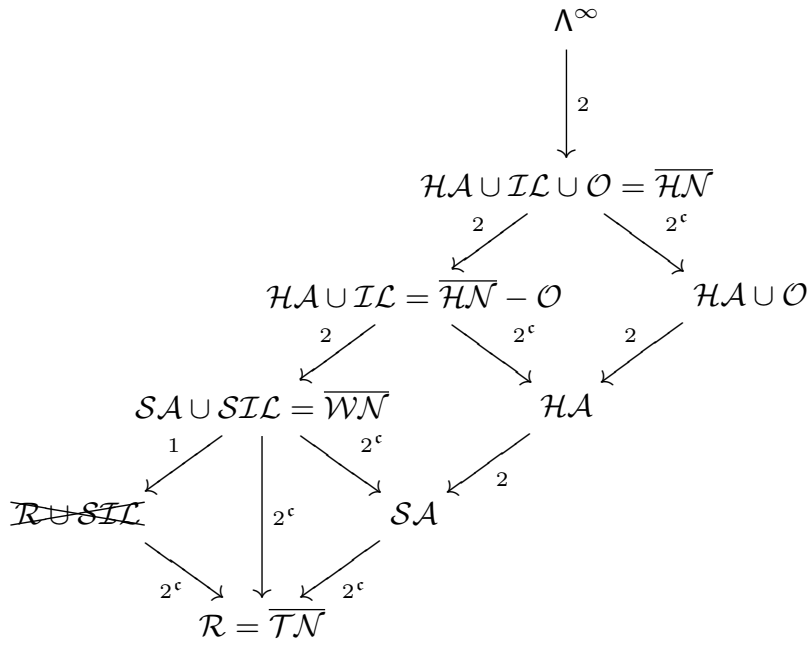

Fig. 1. Lattice of Meaningless Sets extended with an auxiliary vertex. The arrow $U_{1} \stackrel{n}{\rightarrow} U_{2}$ indicates that $U_{1} \supset U_{2}$. The label $n$ shows the cardinality of the class of sets of meaningless terms between $U_{1}$ and $U_{2}$. NB: of all vertices, the vertex $\mathcal{R} \cup \mathcal{S I} \mathcal{L}$ is not a meaningless set.

lambda calculus are precisely the Böhm trees, but now their definition is within the syntax of infinite lambda calculus, whereas [1] needed to develop a special notational machinery. Similarly the choice of the set $\overline{\mathcal{W N}}$ of terms without weak head normal forms as set of meaningless terms leads to the Lévy-Longo trees $[6$, $8,5]$, and the choice for the set $\mathcal{R}$ of rootactive terms recaptures the Berarducci trees $[3,6,8,5]$. Although in the initial papers $[6,8,10,5]$ on infinite lambda calculus only those three sample sets were presented as set of meaningless terms, these sets are not the only sets of meaningless terms. Only in the more recent papers $[14,15,9]$ some aspects of the rich lattice of the sets of meaningless terms have been explored.

The set of all sets of meaningless terms forms a complete lattice as depicted in Figure 1. We say $U_{1} \rightarrow U_{2}$ when $U_{1} \supset U_{2}$. The bottom element is the set $\mathcal{R}$ and the top element is the set $\Lambda^{\infty}$ of finite and $\perp$-free infinite lambda terms. The meet operation $\sqcap$ is intersection and the join $\sqcup$ of two sets of meaningless terms is the smallest meaningless set that contains the two sets.

The purpose of the current paper is to analyse the distribution of the sets of meaningless terms in the lattice by selecting some sets as key vertices and study the cardinality in the intervals between key vertices. The key vertices are all depicted in Figure 1. All key vertices stand for sets of meaningless terms except for the vertex $\mathcal{R} \cup \mathcal{S I L}$. We included this set in the figure to provide a complete picture of the lattice. Because, despite the fact that $\mathcal{R} \cup \mathcal{S I L}$ itself is not meaningless, there are infinitely many sets of meaningless terms between $\mathcal{R}$ and $\mathcal{R} \cup \mathcal{S I} \mathcal{L}$. The other vertices in Figure 1 represent all sets of meaningless 
terms that decompose as the disjoint union of one or more of the basic sets $\mathcal{R}$, $\mathcal{S I} \mathcal{L}, \mathcal{I} \mathcal{L}, \mathcal{S A}, \mathcal{H} \mathcal{A}$ and $\mathcal{O}[15]$

We consider intervals $\left[U_{1}, U_{2}\right]=\left\{U \mid U_{1} \subseteq U \subseteq U_{2}\right.$ and $U$ is meaningless $\}$ between two arbitrary sets $U_{1}$ and $U_{2}$. In particular, we will distinguish between key intervals which are intervals between any two key vertices and elementary key intervals which are key intervals between two consecutive key vertices. We study the cardinality of the elementary key intervals. The cardinalities are shown as labels above the arrows of Figure 1 . Some intervals have cardinality 2 and contain only both extremes. Only one of them has cardinality 1 which is $[\mathcal{R} \cup \mathcal{S I} \mathcal{L}, \mathcal{S} \mathcal{A} \cup$ $\mathcal{S I L}$ ] which contains only the right extreme. All others are uncountable and have cardinality $2^{\mathfrak{c}}$ where $\mathfrak{c}$ is the cardinality of the continuum.

We show that the elementary key intervals with cardinality $2^{\mathfrak{c}}$ cannot be finitely decomposed. In other words, the uncountable intervals cannot be further decomposed as union of finite subintervals. We also study how the key intervals are decomposed as union of elementary key intervals. We prove that all key intervals which are above the set $\mathcal{S} \mathcal{A}$ and $\mathcal{R} \cup \mathcal{S I} \mathcal{L}$ can be decomposed as union of elementary key intervals. For example, $[\mathcal{S} \mathcal{A}, \mathcal{H} \mathcal{A} \cup \mathcal{O}]=[\mathcal{S} \mathcal{A}, \mathcal{H} \mathcal{A}] \cup[\mathcal{H} \mathcal{A}, \mathcal{H} \mathcal{A} \cup \mathcal{O}]$. Not all the key intervals have this nice property. We show that the interval $[\mathcal{R}, \mathcal{S} \mathcal{A} \cup \mathcal{S I L}]$ can not be decomposed as union of elementary key intervals. For this, we show that there are $2^{\mathfrak{c}}$ many sets of meaningless terms which are in $[\mathcal{R}, \mathcal{S} \mathcal{A} \cup \mathcal{S} \mathcal{I} \mathcal{L}]$ and are not in either $[\mathcal{R}, \mathcal{S} \mathcal{A}],[\mathcal{S A}, \mathcal{S} \mathcal{A} \cup \mathcal{S I} \mathcal{L}]$ or $[\mathcal{R}, \mathcal{R} \cup \mathcal{S I} \mathcal{L}]$. This is depicted in Figure 1 by an arrow from $\mathcal{S} \mathcal{A} \cup \mathcal{S I} \mathcal{L}$ to $\mathcal{R}$ labelled by $2^{\mathfrak{c}}$.

We conclude with the observation that the lattice of sets of meaningless sets is neither modular nor distributive. Interestingly, the example translates into a simple counterexample that the lattice of lambda theories is not modular.

\section{Infinitary Lambda Calculus}

We will now briefly recall some notions and facts of infinitary lambda calculus from our earlier work $[6,8,5,13,16]$. We assume familiarity with basic notions and notations from [1]. Let $\Lambda$ be the set of $\lambda$-terms and $\Lambda_{\perp}$ be the set of finite $\lambda$ terms with $\perp$. The set $\Lambda_{\perp}^{\infty}$ of finite and infinite $\lambda$-terms is defined by coinduction from the grammar: $M::=\perp|x|(\lambda x M) \mid(M M)$ where $x$ is a variable from some fixed set of variables $\mathcal{V}$. The set $\Lambda^{\infty}$ is the subset of $\perp$-free terms. The set $\left(\Lambda^{\infty}\right)^{0}$ is the subset of closed terms (without free variables) in $\Lambda^{\infty}$.

We follow the usual conventions on syntax. We will also use the following abbreviations for terms:

$$
\begin{array}{lll}
\mathbf{I}=\lambda x . x & \mathbf{O}=\lambda x_{1} \cdot \lambda x_{2} \cdot \lambda x_{3} \ldots & { }^{\omega} M=(((\ldots) M) M) M \\
\mathbf{K}=\lambda x y . x & \mathbf{\Omega}=(\lambda x \cdot x x)(\lambda x \cdot x x) & \mathbf{F i x}=(\lambda x y \cdot y(x x y))(\lambda x y \cdot y(x x y))
\end{array}
$$

The set $\Lambda_{\perp}^{\infty}$ contains all Böhm, Lévy-Longo and Berarducci trees. These latter notions are usually defined as trees but in the infinitary setting they can equivalently be as terms in $\Lambda_{\perp}^{\infty}$. In $[8,10,5]$ an alternative definition of the set $\Lambda_{\perp}^{\infty}$ is given using a metric. The coinductive and metric definitions are equivalent [2]. 
We define the $\beta$-rule on the set $\Lambda_{\perp}^{\infty}$ of finite and infinite terms:

$$
(\lambda x . M) N \rightarrow M[x:=N]
$$

The reduction $\rightarrow_{\beta}$ is defined as the smallest binary relation containing $\beta$ and closed under contexts. The $\beta_{h}$-reduction is the restriction of the $\beta$-reduction to head redexes. Let $U \subseteq \Lambda^{\infty}$ where $\Lambda^{\infty}$ is the set of terms in $\Lambda_{\perp}^{\infty}$ that do not contain $\perp$. We define the $\perp_{U}$-rule rule on $\Lambda_{\perp}^{\infty}$ as follows:

$$
\frac{M[\perp:=\Omega] \in U \quad M \neq \perp}{M \rightarrow \perp}\left(\perp_{U}\right)
$$

When there is no danger of confusion, we denote $\perp_{U}$ by $\perp$. The reduction $\rightarrow_{\beta \perp_{U}}$ is defined as the smallest binary relation containing $\beta$ and $\perp_{U}$ and closed under contexts. Each set $U$ of meaningless terms gives rise to a different infinitary lambda calculus $\lambda_{U}^{\infty}=\left(\Lambda_{\perp}^{\infty}, \rightarrow_{\beta \perp_{U}}\right)$.

In infinitary lambda calculus we consider strongly converging reduction sequences. These can be of any countable, transfinite length $\alpha: M_{0} \rightarrow_{\rho} M_{1} \rightarrow$ $\ldots M_{\omega} \rightarrow M_{\omega+1} \rightarrow \ldots M_{\omega+\omega} \rightarrow M_{\omega+\omega+1} \rightarrow \ldots M_{\alpha}$, where $\rightarrow$ stands for a $\beta$ - or $\perp$-reduction step. The rough idea is that in such reductions for each limit ordinal $\lambda$, the term $M_{\lambda}$ is defined as the Cauchy limit of the preceding reduction. These limits can then be further reduced. In addition the depth of the contracted redexes goes to infinity at each limit term. We use the following notation: $M \rightarrow N$ denotes a one step reduction from $M$ to $N ; M \rightarrow N$ denotes a finite reduction from $M$ to $N ; M \rightarrow N$ denotes a strongly converging reduction from $M$ to $N$. When $\lambda_{U}^{\infty}$ is confluent and normalising, the normal form of a term $M$ in $\lambda_{U}^{\infty}$ is denoted by $\operatorname{nf}_{U}(M)$. Each confluent and normalising $\lambda_{U}^{\infty}$ gives rise to a $\lambda$-model $\mathfrak{M}_{U}$ and a $\lambda$-theory $\lambda_{U}^{\infty}$ of the finite lambda calculus, as explained in the next definition.

Definition 1. Let $U \subseteq \Lambda^{\infty}$ and $\lambda_{U}^{\infty}$ be confluent and normalising.

1. The $\lambda$-model $\mathfrak{M}_{U}$ induced by the infinitary lambda calculus $\lambda_{U}^{\infty}$ is defined as follows. The domain of $\mathfrak{M}_{U}$ is the set $\mathrm{nf}_{U}(\Lambda)$ of normal forms of finite terms. We interpret a lambda term $M \in \Lambda$ by its normal form $\mathrm{nf}_{U}(M)$ and we define application simply by $\mathrm{nf}_{U}(M) \bullet \mathrm{nf}_{U}(N)=\mathrm{nf}_{U}(M \bullet N)$.

2. The $\lambda$-theory induced by the infinitary lambda calculus $\lambda_{U}^{\infty}$ is denoted by $\mathfrak{T}_{U}$ and defined as $\mathfrak{T}_{U}=\left\{M=N \mid M, N \in \Lambda^{0}\right.$ and $\left.\operatorname{nf}_{U}(M)=\operatorname{nf}_{U}(N)\right\}$.

It is easy to show that $\mathfrak{M}_{U}$ is indeed a $\lambda$-model and $\mathfrak{T}_{U}$ is indeed a $\lambda$-theory of the finite lambda calculus $[1,11]$. Recall that the set of $\lambda$-theories of the finite lambda calculus forms a complete lattice where the meet $\Pi$ is defined by intersection and the join $\sqcup$ of two sets is defined as the smallest theory containing those sets.

We will now define the notion of set of meaningless term. We follow the definition of [9]. This definition differs slightly from the earlier definition in $[8$, $10,5]$ in that the axiom of closure under $\beta$-expansion has been added. This addition has a number of useful consequences. The first is, as observed in [9], 
that with the extra axiom the calculus $\lambda_{U}^{\infty}$ is not only confluent and normalising, but also $\omega$-compressible, just as the three standard instances that give rise to respectively the Böhm, Lévy-Longo and Berarducci trees. The second is that for any set of meaningless terms $U$ the models $\mathfrak{M}_{U}$ and $\mathfrak{M}_{\widehat{U}}$ coincide, where $\widehat{U}$ denotes the expansion $\left\{M \in \Lambda^{\infty} \mid M \rightarrow \prod_{\beta} N \& N \in U\right\}$ of $U$. This is because $\lambda_{U}^{\infty}$ and $\lambda_{\widehat{U}}^{\infty}$ induce the same reduction relations $\rightarrow \beta_{U}$ and $\rightarrow \Perp$. From this follows thirdly that the lambda theories $\lambda_{U}^{\infty}$ and $\lambda_{\widehat{U}}^{\infty}$ are equal. This last consequence is pertinent for the current paper.

Notation 2 Let $M \stackrel{U}{\leftrightarrow} N$ denote that $N$ is obtained from $M$ by replacing some (possibly infinitely many) subterms in $U$ by other terms in $U$.

Definition 3. $[10,5]$ We say that $U \subseteq \Lambda^{\infty}$ is a set of meaningless terms (also called a meaningless set) if it is a set satisfying the axioms of meaninglessness:

1. Rootactiveness. $\mathcal{R}=\left\{M \in \Lambda^{\infty} \mid M\right.$ is rootactive $\} \subseteq U$ (see Definition 5).

2. Closure under $\beta$-reduction. For all $M \in U$, if $M \rightarrow \pi_{\beta} N$ then $N \in U$.

3. Closure under substitution. For all $M \in U, M^{\sigma} \in U$.

4. Overlap. For all $\lambda x . M \in U,(\lambda x . M) N \in U$.

5. Indiscernibility. For all $M, N \in \Lambda^{\infty}, M \in U$ if and only if $N \in U$.

6. Closure under $\beta$-expansion. For all $N \in U$, if $M \rightarrow \pi_{\beta} N$, then $M \in U$.

Note that $\boldsymbol{\Omega} \in U$ for all set $U$ of meaningless terms because $\boldsymbol{\Omega}$ is rootactive. Note also that even without requiring closure under $\beta$-expansion, we have that meaningless sets contain certain $\beta$-expansions: for instance just from rootactiveness and indiscernibility it follows that $\mathbf{I}(\mathbf{I} M) \in U$ and $\mathbf{K} M N \in U$ whenever $M \in U$.

Theorem 4. $[8,10,5]$ If $U$ is a set of meaningless terms then $\lambda_{U}^{\infty}$ is confluent and normalising.

We will now define the sets of meaningless terms that occur in Figure 1. To define these sets, we will first need to introduce new forms of terms analogous to the notions of head, weak head and top normal forms and define certain specific subsets of $\Lambda^{\infty}$ containing the respective forms [15].

Definition 5. We define that

1. $\mathcal{R}=\left\{M \in \Lambda^{\infty} \mid M\right.$ is rootactive $\}$ where $M$ is a rootactive form if for all

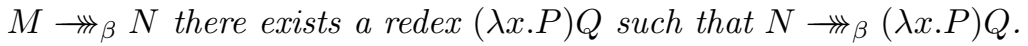

2. $\mathcal{S} \mathcal{A}=\left\{M \in \Lambda^{\infty} \mid M \rightarrow_{\beta} N\right.$ and $N$ is a strong active form $\}$ where $M$ is a strong active form if $M=R P_{1} \ldots P_{k}$ and $R$ is rootactive.

3. $\mathcal{H} \mathcal{A}=\left\{M \in \Lambda^{\infty} \mid M \rightarrow_{\beta} N\right.$ and $N$ is a head active form $\}$ where $M$ is a head active form if $M=\lambda x_{1} \ldots x_{n} . R P_{1} \ldots P_{k}$ and $R$ is rootactive.

4. $\mathcal{S} \mathcal{I}=\left\{M \in \Lambda^{\infty} \mid M \rightarrow \pi_{\beta} N\right.$ and $N$ is a strong infinite left spine form $\}$ where $M$ is a strong infinite left spine form if $M=\left(\ldots P_{2}\right) P_{1}$.

5. $\mathcal{I} \mathcal{L}=\left\{M \in \Lambda^{\infty} \mid M \rightarrow \oiint_{\beta} N\right.$ and $N$ is an infinite left spine form $\}$ where $M$ is an infinite left spine form if $M=\lambda x_{1} \ldots x_{n} \cdot\left(\ldots P_{2}\right) P_{1}$. 
6. $\mathcal{H} \mathcal{N}=\left\{M \in \Lambda^{\infty} \mid M \rightarrow \rightarrow_{\beta} N\right.$ and $N$ is a head normal form $\}$ where $M$ is a head normal form (hnf) if $M=\lambda x_{1} \ldots x_{n} . y P_{1} \ldots P_{k}$.

7. $\mathcal{W N}=\left\{M \in \Lambda^{\infty} \mid M \rightarrow_{\beta} N\right.$ and $N$ is a weak head normal form $\}$ where $M$ is a weak head normal form (whnf) if $M$ is a hnf or $M=\lambda x . N$.

8. $\mathcal{T N}=\left\{M \in \Lambda^{\infty} \mid M \rightarrow \beta N\right.$ and $N$ is a top normal form $\}$ where $M$ is a top normal form (tnf) if it is either a whnf or an application $(N P)$ if there is no $Q$ such that $N \rightarrow \gg_{\beta} \lambda x . Q$.

9. $\mathcal{O}=\left\{M \in \Lambda^{\infty} \mid M \rightarrow \mathbb{H}_{\beta} \mathbf{O}\right\}$.

Theorem 6. $[10,15]$ The sets $\mathcal{R}, \mathcal{S} \mathcal{A}, \mathcal{H} \mathcal{A}, \mathcal{H} \mathcal{A} \cup \mathcal{O}, \mathcal{S} \mathcal{A} \cup \mathcal{S I} \mathcal{L}, \mathcal{H} \mathcal{A} \cup \mathcal{I} \mathcal{L}$, and $\mathcal{H} \mathcal{A} \cup \mathcal{I} \mathcal{L} \cup \mathcal{O}$ are sets of meaningless terms.

As already said in the introduction, by $\overline{\mathcal{H N}}, \overline{\mathcal{W N}}$ and $\overline{\mathcal{T N}}$ we denote the complements in $\Lambda^{\infty}$ of $\mathcal{H N}, \mathcal{W N}$ and $\mathcal{T} \mathcal{N}$ respectively. As it happens, a term is rootactive if and only if it has no top normal form. Hence $\overline{\mathcal{T N}}=\mathcal{R}$.

Definition 7. We define the Berarducci tree of a term $M$ (denoted by $\operatorname{BerT}(M)$ ) by co-recursion as follows.

1. $\operatorname{BerT}(M)=\perp$, if $M$ is rootactive.

2. $\operatorname{BerT}(M)=\lambda x \cdot \operatorname{BerT}(N)$, if $M \rightarrow \beta \lambda x . N$.

3. $\operatorname{BerT}(M)=\operatorname{BerT}(N) \operatorname{BerT}(P)$ if $M \rightarrow_{\beta} N P$ and there is no $Q$ such that $N \rightarrow{ }_{\beta} Q$ and $Q$ is an abstraction.

The Berarducci tree $\operatorname{BerT}(M)$ of a term $M$ is the normal form of $M$ in $\lambda_{U}^{\infty}$ where for $U$ we take $\mathcal{R}[3,8]$.

Definition 8. We define the Lévy-Longo tree of a term $M$ (denoted by $\operatorname{LLT}(M)$ ) by co-recursion as follows.

1. $\operatorname{LLT}(M)=\perp$, if $M$ has no weak head normal form.

2. $\operatorname{LLT}(M)=y \operatorname{LLT}\left(M_{1}\right) \ldots \operatorname{LLT}\left(M_{m}\right)$, if $M \rightarrow_{\beta} y M_{1} \ldots M_{m}$.

3. $\operatorname{LLT}(M)=\lambda x \cdot \operatorname{LLT}(N)$, if $M \rightarrow_{\beta} \lambda x \cdot N$.

The Lévy Longo tree $\operatorname{LLT}(M)$ of a term $M$ is the normal form of $M$ in the calculus $\lambda_{\overline{\mathcal{W N}}}^{\infty}$. It is easy to see that $\overline{\mathcal{W N}}=\mathcal{S} \mathcal{A} \cup \mathcal{S I} \mathcal{L}[8]$.

Definition 9. We define the Böhm tree of a term $M$ (denoted by $\mathrm{BT}(M)$ ) by co-recursion as follows.

1. $\mathrm{BT}(M)=\perp$, if $M$ has no head normal form and

2. $\operatorname{BT}(M)=\lambda x_{1} \ldots \lambda x_{n} . y \mathrm{BT}\left(M_{1}\right) \ldots \mathrm{BT}\left(M_{m}\right)$, if $M$ has a finite $\beta$-reduction to $\lambda x_{1} \ldots \lambda x_{n} . y M_{1} \ldots M_{m}$.

The Böhm tree BT $(M)$ of a term $M$ is the normal form of $M$ in the calculus $\lambda_{\overline{\mathcal{H N}}}^{\infty}$. It is easy to see that $\overline{\mathcal{H} \mathcal{N}}=\mathcal{H} \mathcal{A} \cup \mathcal{I} \mathcal{L} \cup \mathcal{O}[1,8]$.

Notation 10 Let $X \subseteq \Lambda_{\perp}^{\infty}$. We use the following notation: $\operatorname{BerT}(X)=\{\operatorname{BerT}(M) \mid$ $M \in X\}, \operatorname{LLT}(X)=\{\operatorname{LLT}(M) \mid M \in X\}$ and $\mathrm{BT}(X)=\{\mathrm{BT}(M) \mid M \in X\}$. 
Remark 11. Not all (combinations of) basic sets give rise to a meaningless set.

1. The sets $\mathcal{S} \mathcal{I} \mathcal{L}, \mathcal{I} \mathcal{L}$ and $\mathcal{O}$ do not satisfy rootactiveness.

2. The sets $\mathcal{R} \cup \mathcal{S I L}$ and $\mathcal{R} \cup \mathcal{I L}$ do not satisfy indiscernibility: The term ${ }^{\omega} \mathbf{I}=((\ldots) \mathbf{I}) \mathbf{I}$ belongs to both sets $\mathcal{S} \mathcal{L} \mathcal{L}$ and $\mathcal{I} \mathcal{L}$. Since ${ }^{\omega} \mathbf{I}=\left({ }^{\omega} \mathbf{I}\right) \mathbf{I}$, a set satisfying indiscernibility should contain $\boldsymbol{\Omega} \mathbf{I}$ as well. However, $\boldsymbol{\Omega} \mathbf{I}$ does not belong to neither $\mathcal{S} \mathcal{I L}$ nor $\mathcal{I} \mathcal{L}$.

3. The set $\mathcal{R} \cup \mathcal{O}$ is not meaningless. Because any set containing $\mathbf{O}$ must contain $\lambda x . \boldsymbol{\Omega}$ by indiscernibility since $\mathbf{O}=\lambda x . \mathbf{O}$.

Definition 12. 1. The key vertices are the sets that appear in Figure 1, i.e. $\mathcal{R}, \mathcal{S} \mathcal{A}, \mathcal{H} \mathcal{A}, \mathcal{H} \mathcal{A} \cup \mathcal{O}, \mathcal{R} \cup \mathcal{S I L}, \mathcal{S A} \cup \mathcal{S I} \mathcal{L}, \mathcal{H} \mathcal{A} \cup \mathcal{I L}, \mathcal{H} \mathcal{A} \cup \mathcal{O} \cup \mathcal{I} \mathcal{L}$ and $\Lambda^{\infty}$. The set of key vertices is denoted by $\mathcal{K}$.

2. The set $\mathcal{I}_{\mathcal{K}}$ of key intervals is the set of intervals whose extremes are only some of the sets in $\mathcal{K}$, i.e. $\mathcal{I}_{\mathcal{K}}=\left\{\left[U_{1}, U_{2}\right] \mid U_{1}, U_{2} \in \mathcal{K}\right\}$.

3. An elementary key interval is an interval $\left[U_{1}, U_{2}\right]$ in $\mathcal{K}$ such that $U_{1} \subset U_{2}$ and there is no other set $U \in \mathcal{K}$ between $U_{1}$ and $U_{2}$.

The sets in $\mathcal{I}_{\mathcal{K}}$ are all meaningless except for $\mathcal{R} \cup \mathcal{S I L}$. Note that $[\mathcal{S} \mathcal{A}, \mathcal{S} \mathcal{A} \cup$ $\mathcal{S I L}]$ is an elementary key interval, but $[\mathcal{R}, \mathcal{S A} \cup \mathcal{S I L}]$ is not.

\section{The Elementary Key Intervals of Finite Cardinality}

We will now prove that the intervals $[\mathcal{S A}, \mathcal{H} \mathcal{A}],[\mathcal{H A}, \mathcal{H} \mathcal{A} \cup \mathcal{O}],[\mathcal{S} \mathcal{A} \cup \mathcal{S I} \mathcal{L}, \mathcal{H} \mathcal{A} \cup$ $\mathcal{I} \mathcal{L}],[\mathcal{H} \mathcal{A} \cup \mathcal{I} \mathcal{L}, \mathcal{H} \mathcal{A} \cup \mathcal{I} \mathcal{L} \cup \mathcal{O}]$ and $\left[\mathcal{H} \mathcal{A} \cup \mathcal{I} \mathcal{L} \cup \mathcal{O}, \Lambda^{\infty}\right]$ contain only the extremes and have cardinality 2 and the interval $[\mathcal{R} \cup \mathcal{S I} \mathcal{L}, \mathcal{S} \mathcal{A} \cup \mathcal{S I L}]$ has cardinality 1.

Theorem 13. The interval $\left[\overline{\mathcal{H N}}, \wedge^{\infty}\right]$ has cardinality 2.

Proof. By Lemma 45 the only sets in $\left[\overline{\mathcal{H N}}, \Lambda^{\infty}\right]$ are $\overline{\mathcal{H N}}$ and $\Lambda^{\infty}$.

Theorem 14. The intervals $[\mathcal{S A}, \mathcal{H} \mathcal{A}]$ and $[\mathcal{H} \mathcal{A}, \mathcal{H} \mathcal{A} \cup \mathcal{O}]$ have cardinality 2.

Proof. We prove that $\mathcal{H} \mathcal{A}$ is the only meaningless set between $\mathcal{S A}$ and $\mathcal{H} \mathcal{A} \cup \mathcal{O}$. Suppose there exists a set $U$ of meaningless terms such that $\mathcal{S A} \subset U \subset \mathcal{H A} \cup \mathcal{O}$. Then there exists $M \in(\mathcal{H} \mathcal{A} \cup \mathcal{O})-\mathcal{S} \mathcal{A}$. Then $M$ should reduce to a term $N$ either of the form $\mathbf{O}$ or $\lambda x_{1} \ldots x_{n} . R P_{1} \ldots P_{k}$ with $n \geq 1$. By Lemma 46(2), in both cases we have $\mathcal{H} \mathcal{A} \subseteq U$. Since, $U \subset \mathcal{H} \mathcal{A} \cup \mathcal{O}$, we get $U=\mathcal{H} \mathcal{A}$.

Theorem 15. The intervals $[\mathcal{S} \mathcal{A} \cup \mathcal{S} \mathcal{I} \mathcal{L}, \mathcal{H} \mathcal{A} \cup \mathcal{I} \mathcal{L}]$ and $[\mathcal{H} \mathcal{A} \cup \mathcal{I} \mathcal{L}, \mathcal{H} \mathcal{A} \cup \mathcal{I} \mathcal{L} \cup \mathcal{O}]$ have cardinality 2 .

Proof. To prove that $\mathcal{H} \mathcal{A} \cup \mathcal{I L}$ is the only meaningless set between $\mathcal{S} \mathcal{A} \cup \mathcal{S I} \mathcal{L}$ and $\mathcal{H} \mathcal{A} \cup \mathcal{I} \mathcal{L} \cup \mathcal{O}$, we follow the proof of Theorem 14 using Lemma 46(3) instead.

Theorem 16. The interval $[\mathcal{R} \cup \mathcal{S I L}, \mathcal{S} \mathcal{A} \cup \mathcal{I} \mathcal{L}]$ has cardinality 1 .

Proof. It follows from Lemma 47 that the only meaningless set in $[\mathcal{R} \cup \mathcal{S I L}, \mathcal{S} \mathcal{A} \cup$ $\mathcal{I L}]$ is $\mathcal{S A} \cup \mathcal{S I L}$. 
As a consequence of Lemma 46 and Theorems 14, 15 and 47, we have that:

Theorem 17. All the key intervals above $\mathcal{S A}$ and also above $\mathcal{R} \cup \mathcal{S I L}$ can be decomposed as unions of elementary key intervals.

In particular, we have that:

$$
\begin{aligned}
& {\left[\mathcal{S} \mathcal{A}, \Lambda^{\infty}\right]=[\mathcal{S} \mathcal{A}, \mathcal{H} \mathcal{A} \cup \mathcal{I} \mathcal{L} \cup \mathcal{O}] \cup\left[\mathcal{H} \mathcal{A} \cup \mathcal{I} \mathcal{L} \cup \mathcal{O}, \Lambda^{\infty}\right]} \\
& {[\mathcal{S A}, \mathcal{H} \mathcal{A} \cup \mathcal{I} \mathcal{L}]=[\mathcal{S A}, \mathcal{S} \mathcal{A} \cup \mathcal{S} \mathcal{I} \mathcal{L}] \cup[\mathcal{H} \mathcal{A}, \mathcal{H} \mathcal{A} \cup \mathcal{I} \mathcal{L}]} \\
& {[\mathcal{S A}, \mathcal{H} \mathcal{A} \cup \mathcal{I L} \cup \mathcal{O}]=[\mathcal{S A}, \mathcal{S} \mathcal{A} \cup \mathcal{S} \mathcal{I} \mathcal{L}] \cup[\mathcal{H} \mathcal{A}, \mathcal{H} \mathcal{A} \cup \mathcal{I} \mathcal{L}] \cup[\mathcal{H} \mathcal{A} \cup \mathcal{O}, \mathcal{H} \mathcal{A} \cup \mathcal{I} \mathcal{L} \cup \mathcal{O}]} \\
& {[\mathcal{H} \mathcal{A}, \mathcal{H} \mathcal{A} \cup \mathcal{I L} \cup \mathcal{O}]=[\mathcal{H} \mathcal{A}, \mathcal{H} \mathcal{A} \cup \mathcal{I} \mathcal{L}] \cup[\mathcal{H} \mathcal{A} \cup \mathcal{O}, \mathcal{H} \mathcal{A} \cup \mathcal{I L} \cup \mathcal{O}]}
\end{aligned}
$$

\section{The Indecomposable Key Interval $[\mathcal{R}, \mathcal{S} \mathcal{A} \cup \mathcal{S} \mathcal{I} \mathcal{L}]$}

We will show that the key interval $[\mathcal{R}, \mathcal{S} \mathcal{A} \cup \mathcal{S I} \mathcal{L}]$ cannot be decomposed as union of elementary key intervals. For this, we will first show that there are $2^{\mathfrak{c}}$ many sets of meaningless terms in $[\mathcal{R}, \mathcal{S} \mathcal{A} \cup \mathcal{S I L}]-([\mathcal{R}, \mathcal{S} \mathcal{A}] \cup[\mathcal{S} \mathcal{A}, \mathcal{S} \mathcal{A} \cup \mathcal{S I L}] \cup[\mathcal{R}, \mathcal{R} \cup \mathcal{S I} \mathcal{L}])$. As a consequence, we have that

$$
[\mathcal{R}, \mathcal{S} \mathcal{A} \cup \mathcal{S I L}] \neq[\mathcal{R}, \mathcal{S} \mathcal{A}] \cup[\mathcal{S} \mathcal{A}, \mathcal{S} \mathcal{A} \cup \mathcal{S I L}] \cup[\mathcal{R}, \mathcal{R} \cup \mathcal{S I} \mathcal{L}]
$$

We will also show a stronger property which is that the interval $[\mathcal{R}, \mathcal{S} \mathcal{A} \cup \mathcal{S I L}]$ cannot be finitely decomposed, not even by taking intervals with other extremes apart from the sets of Figure 1.

Definition 18. Let $M \in \Lambda^{\infty}$ and $X \subseteq \Lambda^{\infty}$.

1. $M$ is a strong infinite left spine form relative to $X$ (X-sil) if $M=\left((\ldots) P_{2}\right) P_{1}$ and $P_{i} \in X$ for all $i$.

2. $\mathcal{S} \mathcal{I L}_{X}=\left\{M \in \Lambda^{\infty} \mid M \rightarrow_{\beta} N\right.$ and $N$ is a $X$-sil $\}$.

Remark 19. 1. $\mathcal{S} \mathcal{I} \mathcal{L}_{X}$ is not a set of meaningless terms since it does not satisfy rootactiveness. Neither $\mathcal{R} \cup \mathcal{S I} \mathcal{L}_{X}$ is a meaningless set since it does not satisfy indiscernibility. Let $M \in X$. The term ${ }^{\omega} M=((\ldots) M) M \in \mathcal{S} \mathcal{I} \mathcal{L}_{X}$ but $\Omega M$ does not belong to $\mathcal{R} \cup \mathcal{S} \mathcal{I} \mathcal{L}_{X}$.

2. $\mathcal{S} \mathcal{A} \cup \mathcal{S} \mathcal{I} \mathcal{L}_{X}$ is not a meaningless set since it does not satisfy indiscernibility. Consider a term $P \in \Lambda^{\infty}-X$ and $M \in \mathcal{S} \mathcal{I L}_{X}$. The term $\Omega P \in \mathcal{S A}$ but $M P \notin \mathcal{S I} \mathcal{L}_{X}$.

The above remark motivates the following definition:

Definition 20. Let $M \in \Lambda^{\infty}$ and $X \subseteq \Lambda^{\infty}$.

1. $M$ is a strong active form relative to $X$ (X-saf) if $M=R P_{1} \ldots P_{k}$ and $R$ is rootactive and $P_{1}, \ldots, P_{k} \in X$.

2. $\mathcal{S A}_{X}=\left\{M \in \Lambda^{\infty} \mid M \rightarrow_{\beta} N\right.$ and $N$ is a $X$-saf $\}$.

Theorem 21. [9] Let $X \subseteq \operatorname{LLT}\left(\Lambda^{\infty}\right) \cap\left(\Lambda^{\infty}\right)^{0}$. Then, $\mathcal{S} \mathcal{A}_{X} \cup \mathcal{S} \mathcal{I} \mathcal{L}_{X}$ is a set of meaningless terms. 
Corollary 22. There are $2^{\mathfrak{c}}$ many sets of meaningless terms between $\mathcal{R}$ and $\mathcal{S} \mathcal{A} \cup \mathcal{S I} \mathcal{L}$ which are not in either $[\mathcal{R}, \mathcal{S} \mathcal{A}]$, or $[\mathcal{S} \mathcal{A}, \mathcal{S} \mathcal{A} \cup \mathcal{S I L}]$ or $[\mathcal{R}, \mathcal{R} \cup \mathcal{S I L}]$.

Corollary 23. The interval $[\mathcal{R}, \mathcal{S} \mathcal{A} \cup \mathcal{S I L}]$ is not finitely decomposable.

Proof. Clearly, the class $\left\{\mathcal{S} \mathcal{A}_{X} \cup \mathcal{S I} \mathcal{L}_{X} \mid X\right.$ is singleton $\}$ of meaningless sets are all unrelated to each other. They all appear in "parallel intervals".

\section{The Elementary Key Intervals of Infinite Cardinality}

We will now show that the intervals $[\mathcal{R}, \mathcal{S} \mathcal{A}],[\mathcal{R}, \mathcal{R} \cup \mathcal{S I L}],[\mathcal{S} \mathcal{A}, \mathcal{S} \mathcal{A} \cup \mathcal{S} \mathcal{I} \mathcal{L}]$, $[\mathcal{H} \mathcal{A}, \mathcal{H} \mathcal{A} \cup \mathcal{I} \mathcal{L}]$, and $[\mathcal{H} \mathcal{A} \cup \mathcal{O}, \mathcal{H} \mathcal{A} \cup \mathcal{I} \mathcal{L} \cup \mathcal{O}]$ have cardinality $2^{\mathfrak{c}}$ where $\mathfrak{c}$ is the cardinality of the continuum. We can deduce that all these intervals are not finitely decomposable by taking singleton sets as in the proof of Corollary 23 .

\subsection{The interval $[\mathcal{R}, \mathcal{S} \mathcal{A}]$}

We will show that there are $2^{\mathfrak{c}}$ sets of meaningless terms between $\mathcal{R}$ and $\mathcal{S} \mathcal{A}$.

Theorem 24. [15] Let $X \subseteq \operatorname{BerT}\left(\Lambda^{\infty}\right) \cap\left(\Lambda^{\infty}\right)^{0}$. Then, $\mathcal{S} \mathcal{A}_{X}$ is a set of meaningless terms.

Corollary 25. The interval $[\mathcal{R}, \mathcal{S A}]$ has cardinality $2^{\mathfrak{c}}$ and is not finitely decomposable.

\subsection{The interval $[\mathcal{R}, \mathcal{R} \cup \mathcal{S} \mathcal{I} \mathcal{L}]$}

To build a set $U$ of meaningless terms between $\mathcal{R}$ and $\mathcal{S} \mathcal{I} \mathcal{L}$, we have to exclude from $U$ those strong infinite left spines that are prefix of themselves. For instance the assumption $((\ldots) \mathbf{I}) \mathbf{I} \in U$ that would otherwise imply $\boldsymbol{\Omega I} \in U$ (see Remark 11). The set $\mathcal{R} \cup\{((\ldots) \mathbf{I}) \mathbf{I}) \mathbf{K}\}$ is a set of meaningless terms but $\mathcal{R} \cup\{((\ldots) \mathbf{I}) \mathbf{I}\}$ is not.

Definition 26. Let $M \in \Lambda^{\infty}$ and $X, Y \subseteq \Lambda^{\infty}$.

1. $M$ is a strong infinite left spine form relative to $X$ and $Y$ (X, $Y$-silf) if $M=N P$ where $N$ is a strong infinite left spine relative to $X$ and $P \in Y$.

2. $\mathcal{S} \mathcal{L}_{X}^{Y}=\left\{M \in \Lambda^{\infty} \mid M \rightarrow_{\beta} N\right.$ and $N$ is a $X, Y$-silf $\}$.

Theorem 27. Let $X, Y \subseteq \operatorname{LLT}\left(\Lambda^{\infty}\right) \cap\left(\Lambda^{\infty}\right)^{0}$ and $X \cap Y=\emptyset$. Then, $\mathcal{R} \cup \mathcal{S} \mathcal{I} \mathcal{L}_{X}^{Y}$ is a meaningless set.

Corollary 28. The interval $[\mathcal{R}, \mathcal{R} \cup \mathcal{S I L}]$ has cardinality $2^{\mathfrak{c}}$ and is not finitely decomposable. 


\subsection{The interval $[\mathcal{S} \mathcal{A}, \mathcal{S} \mathcal{A} \cup \mathcal{S} \mathcal{I} \mathcal{L}]$}

Let $U$ be a meaningless set. As $\Omega P_{1} \ldots P_{n} \in \mathcal{S} \mathcal{A} \subset U$ we obtain from indiscernibility that $M P_{1} \ldots P_{n} \in U$ for any $M \in U$ and $P_{1}, \ldots P_{n} \in \Lambda^{\infty}$ This motivates:

Definition 29. Let $M \in \Lambda^{\infty}$ and $X \subseteq \Lambda^{\infty}$.

1. $M$ is a segmented strong infinite left spine form relative to $X$ (X-ssf) if there exists a finite set $\left\{P_{1}, \ldots, P_{n}\right\} \subseteq \Lambda_{\perp}^{\infty}$ (possible empty) such that $M=$ $N P_{1} \ldots P_{n}$ and $N$ is a strong infinite left spine relative to $X$.

2. $\mathcal{S S}_{X}=\left\{M \in \Lambda^{\infty} \mid M \rightarrow_{\beta} N\right.$ and $N$ is a $X$-ssf $\}$.

Theorem 30. [9] Let $X \subseteq \operatorname{LLT}\left(\Lambda^{\infty}\right) \cap\left(\Lambda^{\infty}\right)^{0}$. Then, $\mathcal{S} \mathcal{A} \cup \mathcal{S} \mathcal{S}_{X}$ is a meaningless set.

Corollary 31. The interval $[\mathcal{S A}, \mathcal{S} \mathcal{A} \cup \mathcal{S I L}]$ has cardinality $2^{\mathfrak{c}}$ and is not finitely decomposable.

\subsection{The intervals $[\mathcal{H} \mathcal{A}, \mathcal{H} \mathcal{A} \cup \mathcal{I} \mathcal{L}]$ and $[\mathcal{H} \mathcal{A} \cup \mathcal{O}, \mathcal{H} \mathcal{A} \cup \mathcal{I} \mathcal{L} \cup \mathcal{O}]$}

A set $U$ of meaningless terms containing $\mathcal{H A}$ is closed under arbitrary applications and abstractions, i.e. if $M \in U$ and $P_{1}, \ldots P_{n} \in \Lambda^{\infty}$ we should also have that $\lambda x_{1} \ldots x_{k} \cdot M P_{1} \ldots P_{n} \in U$ because $\lambda x_{1} \ldots x_{k} . \Omega P_{1} \ldots P_{n} \in \mathcal{H} \mathcal{A} \subset U$. This motivates the definition:

Definition 32. Let $M \in \Lambda^{\infty}$ and $X \subseteq \Lambda^{\infty}$.

1. $M$ is a segmented infinite left spine form relative to $X(X$-sf) if there exists a finite set $\left\{P_{1}, \ldots, P_{n}\right\} \subseteq \Lambda_{\perp}^{\infty}$ (possible empty) such that $M=$ $\lambda x_{1} \ldots x_{k} \cdot N P_{1} \ldots P_{n}$ and $N$ is a strong infinite left spine relative to $X$.

2. $\mathcal{S}_{X}=\left\{M \in \Lambda^{\infty} \mid M \rightarrow_{\beta} N\right.$ and $N$ is a $X$-sf $\}$.

The first item of the following theorem is as Theorem 47 in [9] but the hypothesis of the second item has been restricted.

Theorem 33. The following sets are sets of meaningless terms:

1. $\mathcal{H} \mathcal{A} \cup \mathcal{S}_{X}$ provided $X \subseteq \operatorname{LLT}\left(\Lambda^{\infty}\right) \cap\left(\Lambda^{\infty}\right)^{0}$.

2. $\mathcal{H} \mathcal{A} \cup \mathcal{O} \cup \mathcal{S}_{X}$ provided $X \subseteq \mathrm{BT}\left(\Lambda^{\infty}\right) \cap\left(\Lambda^{\infty}\right)^{0}$.

Corollary 34. The intervals $[\mathcal{H} \mathcal{A}, \mathcal{H} \mathcal{A} \cup \mathcal{I} \mathcal{L}]$ and $[\mathcal{H} \mathcal{A} \cup \mathcal{O}, \mathcal{H} \mathcal{A} \cup \mathcal{I L} \cup \mathcal{O}]$ have cardinality $2^{\mathfrak{c}}$ and they are not finitely decomposable. 


\section{Non-modularity and non-distributivity}

In this section we prove that the lattice of meaningless sets is neither modular nor distributive by applying the $M_{3}-N_{5}$ Theorem of [4] and the previous theory.

Definition 35. Let $M \in \Lambda^{\infty}$ and $X \subseteq \Lambda^{\infty}$.

1. $M$ is a segmented ${ }^{\omega} \mathbf{K I}$-term relative to $X$ (X-stf) if there exists a finite set $\left\{P_{1}, \ldots, P_{n}\right\} \subseteq X$ (possible empty) such that $M={ }^{\omega} \mathbf{K} \mathbf{I} P_{1} \ldots P_{n}$.

2. $\mathcal{K} \mathcal{I}_{X}=\left\{M \in \Lambda^{\infty} \mid M \rightarrow_{\beta} N\right.$ and $N$ is a $X$-stf $\}$.

Lemma 36. Let $X \subseteq \operatorname{LLT}\left(\Lambda^{\infty}\right) \cap\left(\Lambda^{\infty}\right)^{0}$. Then, $\mathcal{S} \mathcal{A}_{X} \cup \mathcal{K} \mathcal{I}_{X}$ is a meaningless set.

Theorem 37. The lattice of sets of meaningless sets is neither modular nor distributive.

Proof. The key interval $[\overline{\mathcal{T N}}, \mathcal{S} \mathcal{A} \cup \mathcal{S I} \mathcal{L}]$ contains a sublattice isomorphic to $N_{5}$.

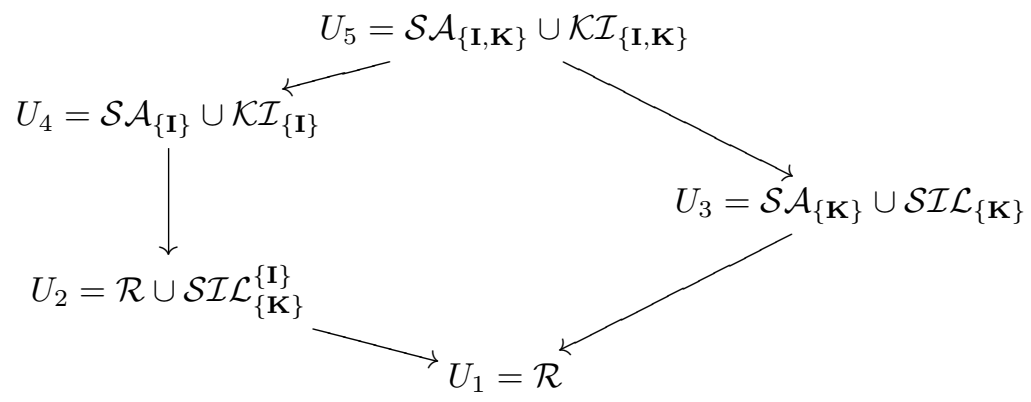

By Theorem 27, $U_{2}=\mathcal{R} \cup \mathcal{S} \mathcal{I} \mathcal{L}_{\{\mathbf{K}\}}^{\{\mathbf{I}\}}$ is the smallest meaningless set closed under $\beta$-expansions containing ${ }^{\omega} \mathbf{K I}$. By Theorem $21, U_{3}=\mathcal{S A}_{\{\mathbf{K}\}} \cup \mathcal{S} \mathcal{I L}_{\{\mathbf{K}\}}$ is the smallest meaningless set closed under $\beta$-expansions containing $\boldsymbol{\Omega K}$ and ${ }^{\omega} \mathbf{K}$. By Theorem 36, $U_{4}=\mathcal{S A}_{\{\mathbf{I}\}} \cup \mathcal{K I}_{\{\mathbf{I}\}}$ is the smallest meaningless set that is closed under $\beta$-expansions and contains $\boldsymbol{\Omega I}$ and ${ }^{\omega} \mathbf{K I}$. By Theorem $36, U_{5}=$ $\mathcal{S A}_{\{\mathbf{I}, \mathbf{K}\}} \cup \mathcal{K} \mathcal{I}_{\{\mathbf{I}, \mathbf{K}\}}$ is the smallest meaningless set closed under $\beta$-expansions containing $\boldsymbol{\Omega I}, \boldsymbol{\Omega K}$ and ${ }^{\omega} \mathbf{K I}$.

To prove that the above five sets form a sublattice of the lattice of sets of meaningless terms, we have to prove that the sublattice is closed under the join and meet operations, i.e. $U_{5}=U_{3} \sqcup U_{4}$ and $U_{1}=U_{2} \sqcap U_{3}$. The latter is trivial because the meet $\Pi$ is intersection. For the first equation, it is not difficult to show that $U_{5}$ is the smallest set of meaningless terms that contains $U_{3}$ and $U_{4}$.

Corollary 38. Let $U_{1}, U_{2}, U_{3}, U_{4}$ and $U_{5}$ be sets of meaningless terms as used in the proof of Theorem $3 \%$.

1. For all $1 \leq i \leq 5$, the infinitary lambda calculus $\lambda_{U_{i}}^{\infty}$ is confluent and normalising. 
2. For all $1 \leq i \leq 5$, the theory $\mathfrak{T}_{U_{i}}$ induced by $\lambda_{U_{i}}^{\infty}$ is consistent.

Part (1) follows from Theorem 4. Part (2) follows from the fact that these five calculi have at least two different normal forms which are $\mathbf{I}$ and $\mathbf{K}$.

In the following lemma, $P^{n}$ denotes the truncation of $P$ at depth $n$.

Lemma 39. Let $U_{2}$ and $U_{3}$ be the sets of meaningless terms used in the proof of Theorem 37. For all $n, P, Q \in \operatorname{BerT}\left(\Lambda_{\perp}^{\infty}\right)$, if $\mathrm{nf}_{U_{2}}(P)=\operatorname{nf}_{U_{2}}(Q)$ and $\mathrm{nf}_{U_{3}}(P)=$ $\mathrm{nf}_{U_{3}}(Q)$ then $P^{n}=Q^{n}$.

Proof. We prove it by induction on $n$. If $n=0$ then $P^{0}=\perp=Q^{0}$. Suppose now that $n>0$. The proof proceeds by cases.

1. Case $P=x P_{1} \ldots P_{k}$. Since $P$ and $Q$ have the same $\mathrm{nf}_{U_{2}}$,

$$
\mathrm{nf}_{U_{2}}(Q)=\mathrm{nf}_{U_{2}}(P)=x \mathrm{nf}_{U_{2}}\left(P_{1}\right) \ldots \mathrm{nf}_{U_{2}}\left(P_{k}\right)
$$

The term $Q$ being in $\beta \perp_{\mathcal{R}}$-normal form can $\beta \perp_{\mathcal{R}}$-reduce to a head normal form only if it is a head normal form itself. Hence, we have that $Q=$ $x Q_{1} \ldots Q_{k}$. Since $P$ and $Q$ have the same $\mathrm{nf}_{U_{2}}$ and the same $\mathrm{nf}_{U_{3}}$, so do $P_{i}$ and $Q_{i}$ for all $1 \leq i \leq k$. Suppose $n>k$. Then,

$$
\begin{aligned}
P^{n} & =x P_{1}^{n-k} \ldots P_{k}^{n-1} \\
& =x Q_{1}^{n-k} \ldots Q_{k}^{n-1} \text { by induction hypothesis } \\
& =Q^{n}
\end{aligned}
$$

Suppose $n \leq k$. Let $i=k-n$. Then,

$$
\begin{aligned}
P^{n} & =\perp P_{i}^{0} \ldots P_{k}^{n-1} \\
& =\perp Q_{i}^{0} \ldots Q_{k}^{n-1} \text { by induction hypothesis } \\
& =Q^{n}
\end{aligned}
$$

2. Case $P=\perp P_{1} \ldots P_{k}$. In this case, we have that $Q=\perp Q_{1} \ldots Q_{k}$ because $U_{2}$ does not contain any head active form. Then, we proceed as in the previous case.

3. Case $P=\lambda x . P_{0}$. In this case, we have that $Q=\lambda x$. $Q_{0}$ because $U_{2}$ does not contain any abstraction. $P_{0}$ and $Q_{0}$ have the same $\mathrm{nf}_{U_{2}}$ and the same $\mathrm{nf}_{U_{3}}$. Then, by induction hypothesis, $P_{0}^{n-1}=Q_{0}^{n-1}$. Hence, $P^{n}=\lambda x \cdot P_{0}^{n-1}=$ $\lambda x \cdot Q_{0}^{n-1}=Q^{n}$.

4. Case $P=\left((\ldots) P_{2}\right) P_{1}$ is a strong infinite left spine. We have two cases:

(a) Case $P=\left(\left(\left(\left({ }^{\omega} \mathbf{K I}\right) P_{k}\right) \ldots\right) P_{2}\right) P_{1}$ for some $k \geq 0$. Since $P$ and $Q$ have the same $\mathrm{nf}_{U_{2}}$ and the same $\mathrm{nf}_{U_{3}}$,

$$
\begin{aligned}
& \operatorname{nf}_{U_{2}}(Q)=\operatorname{nf}_{U_{2}}(P)=\perp \mathrm{nf}_{U_{2}}\left(P_{k}\right) \ldots \operatorname{nf}_{U_{2}}\left(P_{1}\right) \\
& \operatorname{nf}_{U_{3}}(Q)=\mathrm{nf}_{U_{3}}(P)=\perp \mathrm{Iff}_{U_{3}}\left(P_{k}\right) \ldots \mathrm{nf}_{U_{3}}\left(P_{1}\right)
\end{aligned}
$$

This is possible only if $Q=\left({ }^{\omega} \mathbf{K I}\right) Q_{k} \ldots Q_{1}$. Since $P$ and $Q$ have the same $\mathrm{nf}_{U_{2}}$ and the same $\mathrm{nf}_{U_{3}}$, so do $P_{i}$ and $Q_{i}$ for all $1 \leq i \leq k$. Suppose $n \leq k$. Then,

$$
\begin{aligned}
P^{n} & =\perp P_{n}^{0} \ldots P_{1}^{n-1} \\
& =\perp Q_{n}^{0} \ldots Q_{1}^{n-1} \text { by induction hypothesis } \\
& =Q^{n}
\end{aligned}
$$


Suppose $n>k$. Then,

$$
\begin{aligned}
P^{n} & =(\mathbf{K I})^{n-k} P_{k}^{n-k} \ldots P_{1}^{n-1} \\
& =(\mathbf{K I})^{n-k} Q_{k}^{n-k} \ldots Q_{1}^{n-1} \text { by induction hypothesis } \\
& =Q^{n}
\end{aligned}
$$

(b) Otherwise, $P$ is not of the form $\left(\left(\left(\left({ }^{\omega} \mathbf{K I}\right) P_{k}\right) \ldots\right) P_{2}\right) P_{1}$ for any $k \geq 0$. In this case, we have that $Q=\left((\ldots) Q_{2}\right) Q_{1}$ is also a strong infinite left spine because $U_{2}$ does not contain $P$. Since $P$ and $Q$ have the same $\mathrm{nf}_{U_{2}}$ and the same $\mathrm{nf}_{U_{3}}$, so do $P_{i}$ and $Q_{i}$ for all $1 \leq i \leq k$. Then,

$$
\begin{aligned}
P^{n} & =\perp P_{n}^{0} \ldots P_{1}^{n-1} \\
& =\perp Q_{n}^{0} \ldots Q_{1}^{n-1} \text { by induction hypothesis } \\
& =Q^{n}
\end{aligned}
$$

Theorem 40. Let $U_{2}$ and $U_{3}$ be the sets of meaningless terms used in the proof of Theorem 3\%. We have that $\operatorname{nf}_{\mathcal{R}}(M)=\operatorname{nf}_{\mathcal{R}}(N)$ if and only if $\operatorname{nf}_{U_{2}}(M)=$ $\mathrm{nf}_{U_{2}}(N)$ and $\mathrm{nf}_{U_{3}}(M)=\mathrm{nf}_{U_{3}}(N)$.

Proof. $(\Rightarrow)$ Suppose $\operatorname{nf}_{\mathcal{R}}(M)=\operatorname{nf}_{\mathcal{R}}(N)$. Then,

$$
\mathrm{nf}_{U_{2}}(M)=\mathrm{nf}_{U_{2}}\left(\mathrm{nf}_{\mathcal{R}}(M)\right)=\mathrm{nf}_{U_{2}}\left(\mathrm{nf}_{\mathcal{R}}(N)\right)=\mathrm{nf}_{U_{2}}(N)
$$

by Corollary 38 and because $\mathcal{R} \subseteq U_{2}$. Similarly, $\mathrm{nf}_{U_{3}}(M)=\mathrm{nf}_{U_{3}}(N)$.

$(\Leftarrow)$ Suppose $\mathrm{nf}_{U_{2}}(M)=\mathrm{nf}_{U_{2}}(N)$ and $\mathrm{nf}_{U_{3}}(M)=\mathrm{nf}_{U_{3}}(N)$. Let $P=\mathrm{nf}_{\mathcal{R}}(M)=$ $\operatorname{BerT}(M)$ and $Q=\operatorname{nf}_{\mathcal{R}}(N)=\operatorname{BerT}(N)$ (see Definition 7). By Corollary 38 and the fact that $\mathcal{R} \subseteq U_{2}, U_{3}$, we have that

$$
\begin{gathered}
\operatorname{nf}_{U_{2}}(P)=\mathrm{nf}_{U_{2}}(M)=\mathrm{nf}_{U_{2}}(N)=\mathrm{nf}_{U_{2}}(Q) \text { and } \\
\mathrm{nf}_{U_{3}}(P)=\mathrm{nf}_{U_{3}}(M)=\mathrm{nf}_{U_{3}}(N)=\mathrm{nf}_{U_{3}}(Q) .
\end{gathered}
$$

By Lemma $39, P^{n}=Q^{n}$ for all $n$. Hence, $P=Q$.

Corollary 41. $\mathfrak{T}_{U_{2}} \cap \mathfrak{T}_{U_{3}}=\mathfrak{T}_{\mathcal{R}}$.

Theorem 42. Let $U_{3}$ and $U_{4}$ be the sets of meaningless terms used in the proof of Theorem 3\%. We have that $\operatorname{nf}_{\mathcal{R}}(M)=\operatorname{nf}_{\mathcal{R}}(N)$ if and only if $\operatorname{nf}_{U_{3}}(M)=$ $\mathrm{nf}_{U_{3}}(N)$ and $\mathrm{nf}_{U_{4}}(M)=\mathrm{nf}_{U_{4}}(N)$.

The previous theorem is proved similarly to Theorem 41 .

Corollary 43. $\mathfrak{T}_{U_{3}} \cap \mathfrak{T}_{U_{4}}=\mathfrak{T}_{\mathcal{R}}$

The next result is also proved in [12] using a different counterexample.

Theorem 44. The lattice of lambda theories is neither modular nor distributive. 
Proof. The lattice of $\lambda$-theories contains the following sublattice isomorphic to $N_{5}$.

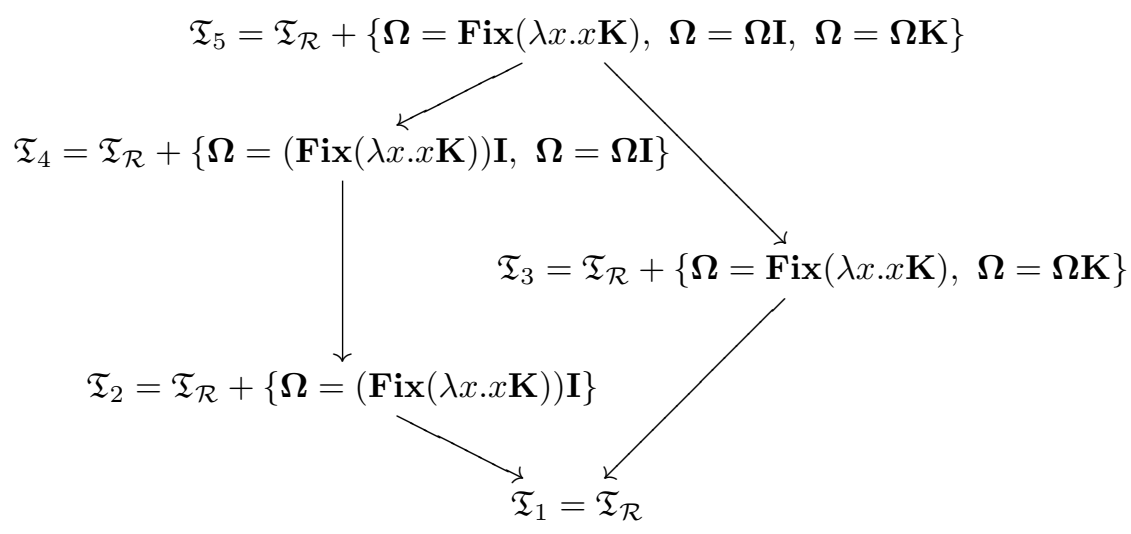

Note that the infinite normal form of $\mathbf{F i x}(\lambda x . x \mathbf{K})$ is ${ }^{\omega} \mathbf{K}$ and the infinite normal form of $(\mathbf{F i x}(\lambda x . x \mathbf{K})) \mathbf{I}$ is ${ }^{\omega} \mathbf{K I}$.

We have that $\left\{\mathfrak{T}_{i} \mid 1 \leq i \leq 5\right\}$ are all consistent because for all $1 \leq i \leq 5$, $\mathfrak{T}_{i} \subseteq \mathfrak{T}_{U_{i}}$ and $\mathfrak{T}_{U_{i}}$ is consistent by Corollary 38 .

To prove that the above five theories form a sublattice of the lattice of $\lambda$ theories, we have to prove that it is closed under the join and meet operations, i.e. $\mathfrak{T}_{5}=\mathfrak{T}_{3} \sqcup \mathfrak{T}_{4}=\mathfrak{T}_{2} \sqcup \mathfrak{T}_{3}$ and $\mathfrak{T}_{1}=\mathfrak{T}_{2} \sqcap \mathfrak{T}_{3}$.

We first prove that $\mathfrak{T}_{5}=\mathfrak{T}_{3} \sqcup \mathfrak{T}_{4}$. It is clear that $\mathfrak{T}_{3}, \mathfrak{T}_{4} \subseteq \mathfrak{T}_{5}$. For any $\mathfrak{T}$ such that $\mathfrak{T}_{3}, \mathfrak{T}_{4} \subseteq \mathfrak{T}$, it is not difficult to prove that $\mathfrak{T}_{5} \vdash M=N$ implies $\mathfrak{T} \vdash M=N$ by induction on the derivation. The derivation rules are the ones of Definition 2.1.4 of [1] extended to include the axioms of $\mathfrak{T}_{\mathcal{R}}, \boldsymbol{\Omega}=\mathbf{F i x}(\lambda x . x \mathbf{K}), \boldsymbol{\Omega}=\boldsymbol{\Omega} \mathbf{I}$ and $\boldsymbol{\Omega}=\boldsymbol{\Omega K}$. Hence, $\mathfrak{T}_{5} \subseteq \mathfrak{T}$ and $\mathfrak{T}_{5}$ is the smallest theory that contains $\mathfrak{T}_{3}$ and $\mathfrak{T}_{4}$. The equality $\mathfrak{T}_{5}=\mathfrak{T}_{2} \sqcup \mathfrak{T}_{4}$ is proved similarly.

We now prove that $\mathfrak{T}_{1}=\mathfrak{T}_{2} \sqcap \mathfrak{T}_{3}$, i.e. $\mathfrak{T}_{1}=\mathfrak{T}_{2} \cap \mathfrak{T}_{3}$. It is clear that $\mathfrak{T}_{1} \subseteq \mathfrak{T}_{2}$ and $\mathfrak{T}_{1} \subseteq \mathfrak{T}_{3}$. Hence, $\mathfrak{T}_{1} \subseteq \mathfrak{T}_{2} \cap \mathfrak{T}_{3}$. On the other hand, we have that $\mathfrak{T}_{1} \cap \mathfrak{T}_{2} \subseteq$ $\mathfrak{T}_{U_{1}} \cap \mathfrak{T}_{U_{2}}=\mathcal{R}$ by Corollary 41 . The proof of the equality $\mathfrak{T}_{1}=\mathfrak{T}_{3} \sqcap \mathfrak{T}_{4}$ is similar using Corollary 43.

\section{Conclusions}

In spite of the fact that the interval $\left[\mathcal{R}, \Lambda^{\infty}\right]$ of all sets of meaningless terms cannot be decomposed as union of elementary key intervals (because of $[\mathcal{R}, \mathcal{S} \mathcal{A} \cup$ $\mathcal{S I L}]$ ), the problem of studying the whole lattice can be reduced to the problem of studying only three intervals: $[\mathcal{R}, \mathcal{S} \mathcal{A} \cup \mathcal{S I L}],[\mathcal{H} \mathcal{A}, \mathcal{H} \mathcal{A} \cup \mathcal{I} \mathcal{L}]$ and $[\mathcal{H} \mathcal{A} \cup$ $\mathcal{O}, \mathcal{H} \mathcal{A} \cup \mathcal{I} \mathcal{L} \cup \mathcal{O}]$. We plan to investigate further what happens in these three intervals. There are far more sets of meaningless terms in these three intervals than the ones shown in this paper. The set $\left\{R M_{1} \ldots M_{2 n} \mid R \in \mathcal{R}, M_{2 i}=\right.$ $\mathbf{I}$ and $\left.M_{2 i+1}=\mathbf{K}\right\}$ is a simple example of a meaningless set in $[\mathcal{R}, \mathcal{S} \mathcal{A}]$ which is 
not of the form $\mathcal{S} \mathcal{A}_{X}$ for any $X$. And we plan to study the relation between the lattice of meaningless sets and the lattice of lambda theories [11].

Acknowledgements. We would like to thank the reviewers for their detailed and helpful comments and suggestions that they provided.

\section{References}

1. H. P. Barendregt. The Lambda Calculus: Its Syntax and Semantics. North-Holland, Amsterdam, Revised edition, 1984.

2. M. Barr. Terminal coalgebras for endofunctors on sets. Theoretical Computer Science, 114(2):299-315, 1999.

3. A. Berarducci. Infinite $\lambda$-calculus and non-sensible models. In Logic and algebra (Pontignano, 1994), pages 339-377. Dekker, New York, 1996.

4. B. A. Davey and H. A. Priestly. Introduction to Lattices and Order. Cambridge University Press, 1990.

5. J. R. Kennaway and F. J. de Vries. Infinitary rewriting. In Terese, editor, Term Rewriting Systems, volume 55 of Cambridge Tracts in Theoretical Computer Science, pages 668-711. Cambridge University Press, 2003.

6. J. R. Kennaway, J. W. Klop, M. R. Sleep, and F. J. de Vries. Infinite lambda calculus and Böhm models. In Rewriting Techniques and Applications, volume 914 of LNCS, pages 257-270. Springer-Verlag, 1995.

7. J. R. Kennaway, J. W. Klop, M. R. Sleep, and F. J. de Vries. Transfinite reductions in orthogonal term rewriting systems. Information and Computation, 119(1):18-38, 1995.

8. J. R. Kennaway, J. W. Klop, M. R. Sleep, and F. J. de Vries. Infinitary lambda calculus. Theoretical Computer Science, 175(1):93-125, 1997.

9. J. R. Kennaway, P. Severi, M. R. Sleep, and F. J. de Vries. Infinitary rewriting: From syntax to semantics. In Processes, Terms and Cycles, volume 3838 of LNCS, pages 148-172. Springer-Verlag, 2005.

10. J. R. Kennaway, V. van Oostrom, and F. J. de Vries. Meaningless terms in rewriting. Journal of Functional and Logic Programming, 1999(1), February 1999.

11. S. Lusin and A. Salibra. The lattice of lambda theories. Journal of Logic and Computation, 14(3):373-394, 2004.

12. A. Salibra. Nonmodularity results for lambda calculus. Fundamenta Informaticae, 45:379-392, 2001.

13. P. Severi and F. J. de Vries. An extensional Böhm model. In Rewriting Techniques and Applications, volume 2378 of LNCS, pages 159-173. Springer-Verlag, 2002.

14. P. Severi and F. J. de Vries. Continuity and discontinuity in lambda calculus. In Typed Lambda Calculus and Applications, volume 3461 of LNCS, pages 369-385. Springer-Verlag, 2005.

15. P. Severi and F. J. de Vries. Order Structures for Böhm-like models. In Computer Science Logic, volume 3634 of LNCS, pages 103-116. Springer-Verlag, 2005.

16. P. Severi and F. J. de Vries. A Lambda Calculus for $D_{\infty}$. Technical report, University of Leicester, 2002. 


\section{A Some Basic Lemmas}

Lemma 45. Let $U \subseteq \Lambda^{\infty}$ satisfy closure under substitution and $\beta$-reduction. If $x P_{1} \ldots P_{n} \in U$ then $U=\Lambda^{\infty}$.

Proof. $M=x P_{1} \ldots P_{n} \in U$. Let $N \in \Lambda^{\infty}$ be arbitrary and $z_{1} \ldots z_{k}$ variables which are not in $N$. By the closure under substitution and reduction axioms, $M\left[x:=\lambda z_{1} \ldots z_{n} \cdot N\right] \rightarrow_{\beta} N \in U$.

Lemma 46. Let $U$ be a meaningless set.

1. If $\lambda x . M \in U$ then $M \in U$.

2. If $\lambda x . M \in U$ then $\mathcal{H} \mathcal{A} \subseteq U$. In particular, if $\mathbf{O} \in U$ then $\mathcal{H} \mathcal{A} \subset U$.

3. If $\lambda x . M \in U$ then $U$ is closed under abstractions, i.e. for all $P \in U$, we have that $\lambda x . P \in U$.

Proof. 1. By the overlap and closure under $\beta$-reduction axioms, $(\lambda x \cdot M) x \rightarrow_{\beta}$ $M \in U$.

2. By the overlap axiom, $(\lambda x . M) Q \in U$ for all $Q \in \Lambda^{\infty}$. By indiscernibility we have that $R Q \in U$ for $R \in \mathcal{R}$ and also $R Q_{1} \ldots Q_{k} \in U$ for all $Q_{i} \in \Lambda^{\infty}$. By the previous part and indiscernibility, $\lambda x . R \in U$ and hence $\lambda x_{1} \ldots x_{n} \cdot R Q_{1} \ldots Q_{k} \in U$.

3. If $\lambda x . M \in U$ then $M \in U$. By indiscernibility, $\lambda x . P \in U$ for any $P \in U$.

Lemma 47. 1. If $\mathcal{S I} \mathcal{L} \subseteq U$ then $\mathcal{S} \mathcal{A} \subseteq U$. Hence, the minimal meaningless set containing $\mathcal{S I L}$ is $\mathcal{S} \mathcal{A} \cup \mathcal{S I} \mathcal{L}$.

2. If $\mathcal{I} \mathcal{L} \subseteq U$ then $\mathcal{H} \mathcal{A} \subseteq U$. Hence, The minimal meaningless set containing $\mathcal{I} \mathcal{L}$ is $\bar{H} \mathcal{A} \cup \mathcal{I} \mathcal{L}$.

Proof. 1. Let $\left.{ }^{\omega} Q=((\ldots) Q) Q\right)$. We have that ${ }^{\omega} Q={ }^{\omega} Q Q \in U$ By indiscernibility, $R Q \in U$ for any $R \in \mathcal{R}$ and also $R Q_{1} \ldots Q_{k} \in U$ for all $Q_{i} \in \Lambda^{\infty}$.

2. $\lambda x_{1} \ldots x_{n} \cdot{ }^{\omega} P Q_{1} \ldots Q_{k} \in U$. By indiscernibility, $\lambda x_{1} \ldots x_{n} . R Q_{1} \ldots Q_{k} \in U$.

As a consequence of the previous lemma, there is no meaningless set between $\mathcal{R} \cup \mathcal{I} \mathcal{L}$ and $\mathcal{H} \mathcal{A} \cup \mathcal{I} \mathcal{L}$ (hence, there is no point in including $\mathcal{R} \cup \mathcal{I} \mathcal{L}$ as a key vertex).

\section{B Checking that a Set is Meaningless}

In this section we show the proof of Theorem 33 part 1 . The rest of the theorems about meaningless sets are proved similarly. We give a criterion for proving that a set is meaningless where indiscernibility has to be checked only on terms whose common structure is a Berarducci tree. Checking this condition on some restricted set of terms will be enough provided the set $U$ is closed under $\beta$ expansions. This criterion differs from the one in [15] on the fact that the common structure is now a Berarducci tree and not a skeleton. 
Definition 48. Let $U \subseteq \Lambda^{\infty}, P, M, N \in \Lambda_{\perp}^{\infty}$.

1. $P \preceq_{U} M$ if $P$ is obtained from $M$ by replacing some subterms of $M$ which belong to $U$ by $\perp$.

2. We say that $P$ is a common structure for $M$ and $N$ relative to $U$ if $P \preceq_{U} M$ and $P \preceq_{U} N$.

E.g. $\perp \perp$ is a common structure for $\Omega \Omega$ and $\Omega(\Omega \Omega)$ with respect to $\mathcal{S} \mathcal{A}$.

Definition 49. [15] The skeleton of a term $M \in \Lambda_{\perp}^{\infty}$ is defined by coinduction.

$$
\begin{array}{ll}
\operatorname{skel}(M)=y & \text { if } M \rightarrow_{\beta} y \\
\operatorname{skel}(M)=\perp & \text { if } M \rightarrow_{\beta} \perp \\
\operatorname{skel}(M)=\lambda x \cdot \operatorname{skel}(N) & \text { if } M \rightarrow_{\beta} \lambda x . N \\
\operatorname{skel}(M)=\operatorname{skel}(N) \operatorname{skel}(P) & \text { if } M \rightarrow_{\beta} N P \text { and there is no } Q \text { such that } N \rightarrow_{\beta} \lambda x . Q \\
\operatorname{skel}(M)=M & \text { if } M \text { does not have a top normal form }
\end{array}
$$

The skeleton of a term is essentially the Berarducci tree of a term but instead of replacing rootactive terms by $\perp$, we leave rootactive terms untouched.

Lemma 50. Let $M \in \Lambda_{\perp}^{\infty}$. Then $M \rightarrow_{\beta}$ skel $(M)$ and skel $(M)$ is either a head normal form, $\perp P_{1} \ldots P_{k}$, a head active form, an infinite left spine or $\mathbf{O}$.

Note that $\operatorname{BerT}(M)=\operatorname{BerT}(\operatorname{skel}(M)) \preceq_{U} \operatorname{skel}(M)$ for any set $U \supseteq \mathcal{R}$.

Lemma 51. Let $U$ be closed under substitution. If $M \preceq_{U} N$ and $M \rightarrow_{\beta} M^{\prime}$ then $N \rightarrow_{\beta} N^{\prime}$ and $M^{\prime} \preceq_{U} N^{\prime}$ for some $N^{\prime}$.

Proof. This is proved by induction on the length of the reduction sequence.

If the set $U$ contains abstractions, from $M \preceq_{U} N$ and $N \rightarrow_{\beta_{h}} N^{\prime}$, we may not be able to find $M^{\prime}$ such that $M \rightarrow_{\beta_{h}} M^{\prime}$ and $M^{\prime} \preceq_{U} N^{\prime}$. For example, suppose $U$ contains $\lambda x . \boldsymbol{\Omega}$. Then, $\perp \mathbf{I} \preceq_{U}(\lambda x . \boldsymbol{\Omega}) \mathbf{I} \rightarrow_{\beta_{h}} \boldsymbol{\Omega}$ but $\perp \mathbf{I}$ cannot be obtained from $\Omega$ by replacing terms in $U$ by $\perp$.

Lemma 52. Let $U$ be closed under substitution and $\beta$-reduction. If $M \preceq_{U} N$ and $N \rightarrow_{\beta_{h}} N^{\prime}$, then we have two cases:

1. $M \rightarrow_{\beta_{h}} M^{\prime}$ and $M^{\prime} \preceq_{U} N^{\prime}$ for some $M^{\prime}$,

2. $M \rightarrow_{\beta_{h}} \lambda x_{1} \ldots x_{k} \cdot \perp Q_{1} \ldots Q_{n}$ with $n \geq 1$ and $U$ contains some abstraction.

Proof. We prove it for one step of $\beta_{h}$-reduction. Suppose

$$
\begin{aligned}
N & =\lambda x_{1} \ldots x_{k} \cdot\left(\lambda x \cdot Q_{0}\right) Q_{1} \ldots Q_{n} \text { and } \\
N^{\prime} & =\lambda x_{1} \ldots x_{k} \cdot Q_{0}\left[x:=Q_{1}\right] Q_{2} \ldots Q_{n} .
\end{aligned}
$$

Then we have four cases:

1. $M=\lambda x_{1} \ldots x_{i}$. $\perp$. Then $M \preceq_{U} N^{\prime}$.

2. $M=\lambda x_{1} \ldots x_{k} \cdot \perp Q_{1} \ldots Q_{n}$. This case is possible only if $U$ contains the abstraction $\lambda x . Q_{0} \in U$ which has been replaced by $\perp$. 
3. $M=\lambda x_{1} \ldots x_{k} \cdot\left(\lambda x \cdot Q_{0}^{\prime}\right) Q_{1}^{\prime} \ldots Q_{n}^{\prime}$ with $Q_{i}^{\prime} \preceq_{U} Q_{i}$ for all $0 \leq i \leq n$. Then

$$
\begin{aligned}
M^{\prime}=\lambda x_{1} \ldots x_{k} \cdot Q_{0}^{\prime}\left[x:=Q_{1}^{\prime}\right] Q_{1} \ldots Q_{n}^{\prime} & \preceq_{U} N^{\prime} \\
& =\lambda x_{1} \ldots x_{k} \cdot Q_{0}\left[x:=Q_{1}\right] Q_{1} \ldots Q_{n}
\end{aligned}
$$

This is because $U$ is closed under substitutions and we have that $Q_{0}^{\prime}[x:=$ $\left.Q_{1}\right] \preceq_{U} Q_{0}\left[x:=Q_{1}\right]$.

4. $M=\perp Q_{i}^{\prime} \ldots Q_{n}^{\prime}$ for $2 \leq i \leq n$. In this case $\left(\lambda x \cdot Q_{0}^{\prime}\right) Q_{1}^{\prime} \ldots Q_{i-1}^{\prime} \in U$ has been replaced by $\perp$. Since $U$ is closed under $\beta$-reduction, $Q_{0}^{\prime}\left[x:=Q_{1}^{\prime}\right] \ldots Q_{i-1}^{\prime} \in U$ and hence $M \preceq_{U} Q_{0}^{\prime}\left[x:=Q_{1}^{\prime}\right] \ldots Q_{n}^{\prime}$.

Lemma 53. Let $U$ be closed under substitution and $\beta$-reduction. If $M \preceq_{U} N$ and $M$ rootactive then $N$ is rootactive.

Proof. Suppose $N$ is not rootactive. Then $N \rightarrow_{\beta_{h}} N^{\prime}$ and $N^{\prime}$ is a top normal form. By Lemma 52, we have two cases

1. either $M \rightarrow_{\beta_{h}} \lambda x_{1} \ldots x_{k} \cdot \perp Q_{1} \ldots Q_{n}$. Since $M$ is rootactive, this case is not possible.

2. or we have that there exists $M^{\prime}$ such that $M \rightarrow_{\beta_{h}} M^{\prime}$ and $M^{\prime} \preceq_{U} N^{\prime}$. If $N^{\prime}$ is a head normal form or an abstraction, so is $M^{\prime}$. Then, these cases are not possible because $M$ is rootactive. Now, suppose that $N^{\prime}$ is an application of the form $N_{1} N_{2}$ where $N_{1}$ does not reduce to an abstraction. Then $M^{\prime}=$ $M_{1} M_{2}$ with $M_{1} \preceq_{U} N_{1}$ and $M_{2} \preceq_{U} N_{2}$. Suppose towards a contradiction that $M_{1}$ reduces an abstraction $\lambda x . M_{0}$. By Lemma $51, N_{1}$ reduces to a term $N_{3}$ such that $\lambda x \cdot M_{0} \preceq_{U} N_{3}$. Then, $N_{3}$ should be an abstraction as well.

Lemma 54. Let $U$ satisfy rootactiveness, be closed under substitution and $\beta$ reduction. Let $P$ be a skeleton, i.e. $\operatorname{skel}(P)=P$. If $P \preceq_{U} M$ then $\operatorname{BerT}(P) \preceq_{U}$ $M$.

Proof. $\operatorname{BerT}(P)$ is obtained from $P$ by replacing all the rootactive subterms of $P$ by $\perp$. We prove that $(\operatorname{BerT}(P))^{n} \preceq_{U} M^{n}$ for all $n$ where $M^{n}$ denotes the truncation of $M$ at depth $n$. Since $(\operatorname{BerT}(P))^{n}$ is finite, we can proceed by induction on the number of symbols of $(\operatorname{BerT}(P))^{n}$. We show only the case when $P=P_{0} \ldots P_{k}$ and $P_{0}$ is rootactive. Then $M=M_{0} \ldots M_{k}$ and $P_{i} \preceq_{U} M_{i}$ for $0 \leq i \leq k$. By Lemma 53, $M_{o}$ is rootactive. Since $M_{0}$ does not contain $\perp$ 's, we have that $\perp \preceq_{U} M_{0}$. By Induction Hypothesis, $\left(\operatorname{BerT}\left(P_{i}\right)\right)^{n} \preceq_{U} M_{i}^{n}$ for $1 \leq i \leq k$. Hence, $(\operatorname{Ber} T(P))^{n} \preceq_{U} M^{n}$.

Definition 55. Let $U \subseteq \Lambda^{\infty}$. We say that $U$ satisfies the axiom of weak indiscernibility if for all $P \in \operatorname{BerT}\left(\Lambda_{\perp}^{\infty}\right)$ such that $P \preceq_{U} M$ and $P \preceq_{U} N$, we have that $M \in U$ if and only if $N \in U$.

Theorem 56. Suppose $U \subset \Lambda^{\infty}$ satisfies: closure under $\beta$-reduction, closure under $\beta$-expansion, closure under substitution, rootactiveness and weak indiscernibility. Then $U$ satisfies indiscernibility. If in addition $U$ satisfies overlap, then $U$ is a meaningless set. 
Proof. We prove indiscernibility. Let $M \stackrel{U}{\leftrightarrow} N$. Then there exists $P$ such that $P \preceq_{U} M$ and $P \preceq_{U} N$. By Lemma 50 and Lemma 51, we have that skel $(P) \preceq_{U}$ $M^{\prime}$ and $\operatorname{skel}(P) \preceq_{U} N^{\prime}$ for some $M^{\prime}, N^{\prime}$ such that $M \dddot{\#}_{\beta} M^{\prime}$ and $N \rightarrow \dddot{\#}_{\beta}$ $N^{\prime}$. By Lemma 54, BerT $(\operatorname{skel}(P)) \preceq_{U} M^{\prime}$ and $\operatorname{BerT}(\operatorname{skel}(P)) \preceq_{U} N^{\prime}$. By weak indiscernibility, $M^{\prime} \in U$ if and only if $N^{\prime} \in U$. Since $U$ is closed under $\beta$ reduction and $\beta$-expansion, we have that $M \in U$ if and only if $N \in U$.

The following lemma will be used in the next proof of Theorem 33 part 1 .

Lemma 57. Suppose $U$ satisfies the first four axioms of meaningless. Let $P \in$ $\operatorname{BerT}\left(\Lambda_{\perp}^{\infty}\right)$ and $P \preceq_{U} M$. If $M \rightarrow_{\beta} M^{\prime}$ and $M^{\prime}$ does not contain any subterm in $U$ then $P=M=M^{\prime}$.

Proof. This is proved by induction on the length of the reduction sequence $M \dddot{m}_{\beta} M^{\prime}$. We prove the case when the length is 1 . Let $M=C\left[\left(\lambda x \cdot M_{0}\right) M_{1}\right]$ and $M^{\prime}=C\left[M_{0}\left[x:=M_{1}\right]\right]$. Since $M^{\prime}$ does not have any subterm in $U$, we have that $M_{0}\left[x:=M_{1}\right] \notin U$. By closure under substitutions, $M_{0} \notin U$. By overlapping $\left(\lambda x . M_{0}\right)$ and $\left(\lambda x . M_{0}\right) M_{1} \notin U$. Then $P$ should contain a $\beta$-redex of the form $\left(\lambda x . P_{0}\right) P_{1}$ where $P_{0} \preceq_{U} M_{0}$ and $P_{1} \preceq_{U} M_{1}$. But this contradicts the fact that $P$ is in $\beta \perp$-normal form.

We now prove Theorem 33 part 1.

Proof. We apply Theorem 56. We have to prove weak indiscernibility for $U=$ $\mathcal{H} \mathcal{A} \cup \mathcal{S}_{X}$. Suppose $P \in \operatorname{BerT}\left(\Lambda_{\perp}^{\infty}\right)$ and $P \preceq \mathcal{H} \mathcal{A} \cup \mathcal{S}_{X} M, N$.

1. If $P$ is either a head normal form or $\mathbf{O}$, so are $M$ and $N$. Hence, $M, N \notin$ $\mathcal{H} \mathcal{A} \cup \mathcal{S}_{X}$.

2. Suppose $P=\lambda x_{1} \ldots \lambda x_{n} \cdot \perp P_{1} \ldots P_{k}$ is a head bottom form. Then,

$$
M=\lambda x_{1} \ldots x_{n} \cdot M_{o} M_{1} \ldots M_{k} \text { and } N=\lambda x_{1} \ldots x_{n} \cdot N_{0} N_{1} \ldots N_{k}
$$

where $M_{o}, N_{0} \in \mathcal{H} \mathcal{A} \cup \mathcal{S}_{X}$ and $P_{i} \preceq \mathcal{H} \mathcal{A} \cup \mathcal{S}_{X} M_{i}, N_{i}$ for $0 \leq i \leq k$. We have two options for $N_{0}$. If $N_{0} \in \mathcal{H} \mathcal{A}$ then $N \in \mathcal{H} \mathcal{A}$. And if $N_{0} \in \mathcal{S}_{X}$ then $N \in \mathcal{S}_{X}$.

3. Suppose $P=\lambda x_{1} \ldots x_{n}$. ((..) $\left.P_{2}\right) P_{1}$ is an infinite left spine. Then,

$$
M=\lambda x_{1} \ldots x_{n} \cdot\left((\ldots) M_{2}\right) M_{1} \text { and } N=\lambda x_{1} \ldots x_{n} \cdot\left((\ldots) N_{2}\right) N_{1}
$$

where $P_{i} \preceq \mathcal{H} \mathcal{A} \cup \mathcal{S}_{X} M_{i}, N_{i}$ for all $i$. If $M \in \mathcal{S}_{X}$ then there exists $l$ such that for all $m \geq l, M_{m}$ reduces to a Lévy Longo tree without $\perp$. Hence $\operatorname{LLT}\left(M_{m}\right)$ does not contain any subterm in $\mathcal{H} \mathcal{A} \cup \mathcal{S}_{X}$. By Lemma 57 , we have that $\operatorname{LLT}\left(M_{m}\right)=M_{m}=P_{m}$. Since $P_{m}$ does not contain $\perp$ 's, we also have that $M_{m}=P_{m}=N_{m}$. Then, $N \in \mathcal{S}_{X}$. 